\author{
MATERIALS SCIENCE AND ENGINEERING A \\ Professor Enrique J Lavernia, Editor \\ Chemical and Biochemical Engineering and Materials Science \\ University of California, Irvine \\ Irvine, CA 92697-2540, USA
}

Letter to the editor of MATERIALS SCIENCE AND ENGINEERING A
Reference: EL2001-261

\title{
The importance of experimental design
}

Letter concerning the paper:

Ji CH, Loh NH, Khor KA, Tor. Sintering study of 316L stainless steel metal injection

molding parts using Taguchi method: final density. SB311: (1-2) 74-82 JUL 312001

In the paper, Ji, Loh, Khor and Tor have performed an experiment in the metal injection molding process, where their goal has been to predict the density of the sintered specimens as a function of different processing conditions. In an effort to reduce experimental labour, the researchers have used the so-called Taguchi method to select the experimental design and partly to analyse the experiment. In order to maximize the sintered density, the authors conclude that an optimal setting of the tested factors includes an intermediate sintering temperature of $1250{ }^{\circ} \mathrm{C}$, a fast heating rate of the furnace of $20^{\circ} \mathrm{C} / \mathrm{min}$, the longest tested sintering time of 90 minutes and that vacuum sintering is most advantageous. The recommended settings are then verified with a verification experiment. As much as I appreciate the researchers' effort to maximize information output from an experiment I believe that they took a large risk in using the Taguchi concept, and this has perhaps led to erroneous conclusions of how the process should be set optimally.

In the experiment, the authors have used an experimental array often recommended by Taguchi, called $\mathrm{L}_{9}$, which if no interactions are present may reveal if the four experimental factors have an effect on the resulting density, and also if non-linear relations between experimental factors and the response exists. The selected factors are sintering temperature, heating rate, sintering time, and sintering atmosphere. A factor has to be tested in at least three levels to spot non-linear relations between the factor and the response, so to test all setting combinations for four factors and to do this in three levels for each factor in a full factorial experiment would require $3^{4}=81$ runs. The $\mathrm{L}_{9}$-design with only nine runs thus represents an attractive alternative for the experimental budget. As seen from Figure 1, this in turn leads to the conclusion that the authors have assumed that no interaction effects present. An assumption that interactions are not important to test suggests, for instance that the density effect resulting from changing temperature is the same regardless of the setting of the sintering time or regardless if the sintering is performed in vacuum or in nitrogen. Heating rate is not supposed to affect the temperature effect either. Although these and other assumptions might be true, I believe that they are quite bold statements. A better way to design an experiment would be to list the possible interactions and then test these as well. 


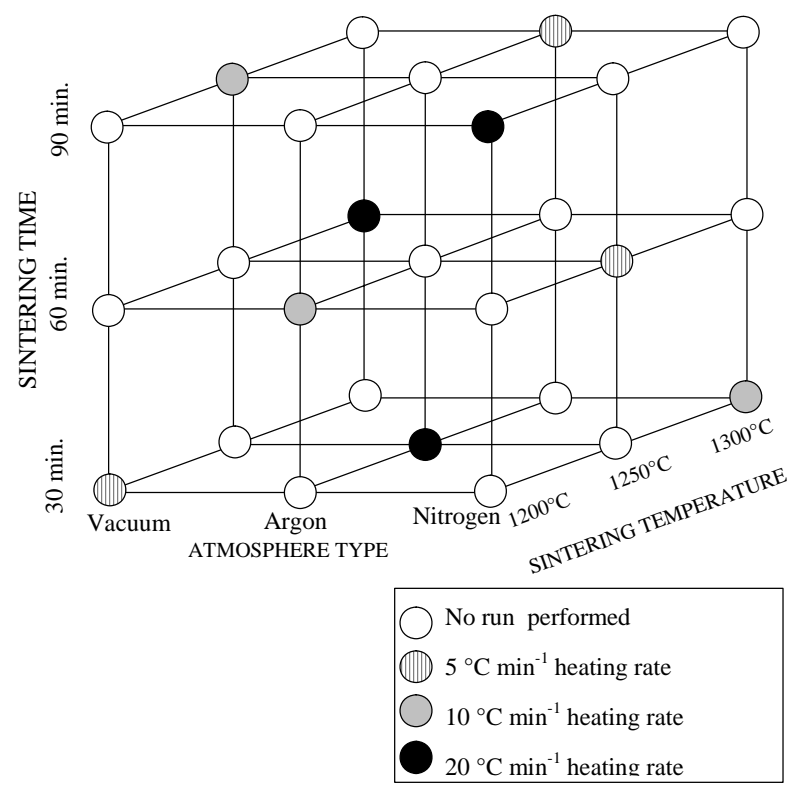

Figure 1. Experimental design of experiment. Each circle represents the experimental conditions of a run. Filled or textured circles represent design points that were run in the study.

Looking at the results, the largest effect comes from using vacuum sintering instead of argon or nitrogen. Vacuum reduces internal pore pressure as well as acts as a reducing agent to reduce surface oxides, so this is to be expected. Normally, I would expect faster densification and higher total density as sintering temperature is raised. This was not found, but the optimum setting was selected to $1250{ }^{\circ} \mathrm{C}$, the intermediate level. The selection was based on the temperature effect, which in turn primarily was based on the large differences between vacuum sintering at $1200^{\circ}$ and sintering at higher temperatures. Of all sintering runs, it was only for vacuum sintering that the highest density was obtained at $1250^{\circ} \mathrm{C}$, and the effect was small. Naturally, one cannot draw conclusions from such comparisons without taking the settings of other factors into account, as well as considering the experimental variation. It is quite possible that the non-linearity of the temperature effect is just a confounding of an interaction, with, for instance sintering time. The sintering time during the highest density runs was 90 minutes and for the $1300^{\circ} \mathrm{C}$ vacuum sintering only 60 minutes. This may well have explained the small density difference between these runs. During the $1200^{\circ} \mathrm{C}$ run, sintering time was only 30 minutes. My guess is that the linear effects of temperature and sintering time is real but small, and that there exists an interaction between them making sintering at low temperature together with short sintering times unwanted, but as densification becomes increasingly difficult as the pores close and coalesque, their combined effect drops. Non-linear effects may also be present, but they are probably much smaller than linear main factor and two-factor interaction effects. Unfortunately, with selected experimental design, this can only be a speculation.

From the paper, it is not clear if the replicated tests are true replicates or if, in fact four specimens were sintered at once. If the replicates are true replicates, the number of test runs should total to 36. With as many runs as that, much more information could be gathered without loss of 
experimental certainty if a reduced factorial experiment was used. If the test replicates were just four specimens sintered at the same time, the density variation between the samples does not represent the total variation of the experiment and cannot be used for statistical tests. By sintering all samples at once any error in setting the sintering temperature, normal process fluctuation of atmosphere chemistry and processing time etc. are unaccounted for. The between-samplevariations should therefore be smaller than the total experimental variation. The measured variation is then a measure of furnace differences due to different placements of the sample or variations during production of the green parts. Nothing wrong with this, but then the resulting variation should not be used for predictions for follow-up runs, nor is it possible to know how large the effects should be in order to classify them as active.

One reason for the use of the Taguchi method instead of classical design of experiments given by the authors is that Montgomery [1] supposedly has argued that the technique should be more flexible and versatile than classical design of experiments. I have not found that statement in the book, but Montgomery describes the Taguchi methods as "unnecessary complicated, inefficient and sometimes ineffective" [2]. I think that the problems with the experimental evaluation of the mentioned experiment are examples of why Montgomery felt this way.

1. D.C. Montgomery, Design and Analysis of Experiments, third ed., Wiley, New York, 1991

2. Ibid, page 415.

\author{
Sincerely, \\ Bjarne Bergquist, $\mathrm{PhD}$ \\ Assistant professor \\ Division of Quality Technology and Statistics \\ Luleå University of Technology \\ SE 97187 Luleå \\ Sweden \\ E-mail: Bjarne.Bergquist@ies.luth.se
}

